\title{
A pedicled autologous choroid RPE patch: a technique to preserve perfusion
}

\author{
Marcin Stopa, Jarosław Kocięcki, Piotr Rakowicz, Andrzej Dmitriew \\ Department of Ophthalmology, Poznan University of Medical Sciences, Poland
}

Videosurgery Miniinv 2012; 7 (3): 220-223

DOI: $10.5114 /$ wiitm.2011.28910

\begin{abstract}
The aim of the study is to report a technique of a pedicled autologous choroid retinal pigment epithelium (RPE) patch that aims to preserve perfusion of the transplanted tissue. A case report of a patient with sudden vision deterioration due to submacular hemorrhage in age-related macular degeneration. The surgery involved a 180-degree peripheral retinectomy and the creation of a pedicled graft instead of an isolated one. Outcome measures included preoperative and postoperative visual acuity and optical coherence tomography scans at 1, 3, 6, 12 months and patch vascularization on postoperative indocyanine green angiography. Postoperatively the patch was positioned under the fovea with an intact pedicle. Indocyanine green angiography showed perfusion through the pedicle and patch vasculature on the third postoperative day. Best corrected visual acuity improved from 0.5/50 to 5/50 at 1 month and remained stable over 1 year follow-up. No choroidal neovascularization recurrence was observed. This case report demonstrates the feasibility of a pedicled RPE-choroid graft that is an alternative to a free isolated graft. Our modification of patch surgery, by demonstrating early perfusion, offers an advantage, similar to macular translocation, when photoreceptors are embedded in RPE and choroid with blood circulation immediately after the surgery.
\end{abstract}

Key words: retinal pigment epithelium choroid patch, age-related macular degeneration, vitrectomy.

\section{Introduction}

Vision loss in age-related macular degeneration (AMD) is the result of choroidal neovascularization (CNV) [1]. In autologous retinal pigment epithelium (RPE)-choroid transplantation surgery healthy subretinal structures are moved to a subfoveal site after CNV removal. Recent reports have demonstrated some success in improving vision in eyes with submacular hemorrhage [2], RPE tear [3] and not responding to antivascular endothelial growth factor (VEGF) therapy [4].

So far the surgical technique including all its variations has almost invariably consisted of a complete separation of the graft from the surrounding choroid. After patch translocation to the new location the re- vascularization of initially non-perfused graft has to be reestablished. However, some of the isolated patches do not become revascularized and fail to regain their function [5-7].

Plastic and reconstructive surgeons are familiar with treatment of skin defects with pedicle flaps [8]. The method of closing soft tissue defects has the advantage of maintaining perfusion to the graft through the pedicle containing major vascular branches.

In this report we describe a technique of autologous RPE choroid transplantation that involves creation of a pedicled RPE choroid flap (Figure 1) that is analogous to the aforementioned method known in plastic and reconstructive surgery. 


\section{Case report}

The feasibility of the technique is presented in a case report of a 75-year-old female patient. She was referred to our institution with a sudden onset central vision loss in her left eye 2 weeks earlier. The patient had been diagnosed as having age-related macular degeneration, but did not receive any form of therapy. Her initial visual acuity was $0.5 / 50$ and had a large 17 DA (disc area) submacular hemorrhage on fundoscopy and angiography and a dome-shaped hemorrhagic sub-RPE elevation and RPE tear with subretinal fluid on optical coherence tomography (OCT) (Figure 2). The vision in the fellow eye was 5/25 with numerous drusen in the macula. The patient gave written informed consent regarding her knowledge of the treatment procedure. The study was approved by the Ethical Committee of Poznan University of Medical Sciences.

She underwent a 20-gauge 3-port pars plana vitrectomy. The initial steps of the surgery were performed by one vitreoretinal surgeon (MS) as described previously [2, 4]. After peripheral temporal $180^{\circ}$ retinotomy was performed, the retina was folded nasally and perfluorocarbon liquid (PFCL) was injected into the subretinal space. The submacular hemorrhage and CNV complex were removed. An area of the full thickness pedicled patch of choroid, choriocapillaris, Bruch's membrane and RPE from the superior paramacular uveal bed was marked using the argon laser. Thereafter the graft was dissected with vertical retinal scissors leaving out an intact pedicle. Choroidal bleeding was controlled by endocautery and/or elevation of intraocular pressure. The graft was pulled bimanually with two forceps in a circular fashion to the subfoveal area (Figure 1). During this maneuver the pedicle became slightly folded. Perfluorocarbon liquid was aspirated from the subretinal space and transferred onto the unfolded retina. Peripheral laser photocoagulation was performed at the edge of the retinotomy and a direct PFCL silicone oil 1000 exchange was carried out with a back flush flute needle. The silicone oil was removed 12 weeks later.

Preoperative examination included best corrected visual acuity (BCVA), dilated fundoscopy, OCT and indocyanine green (ICG) angiography. Similarly, on each postoperative visit at 1, 3, 6 and 12 months, BCVA and dilated fundoscopy and OCT were performed. The ICG angiography was carried out to assess graft perfusion on the third postoperative day.

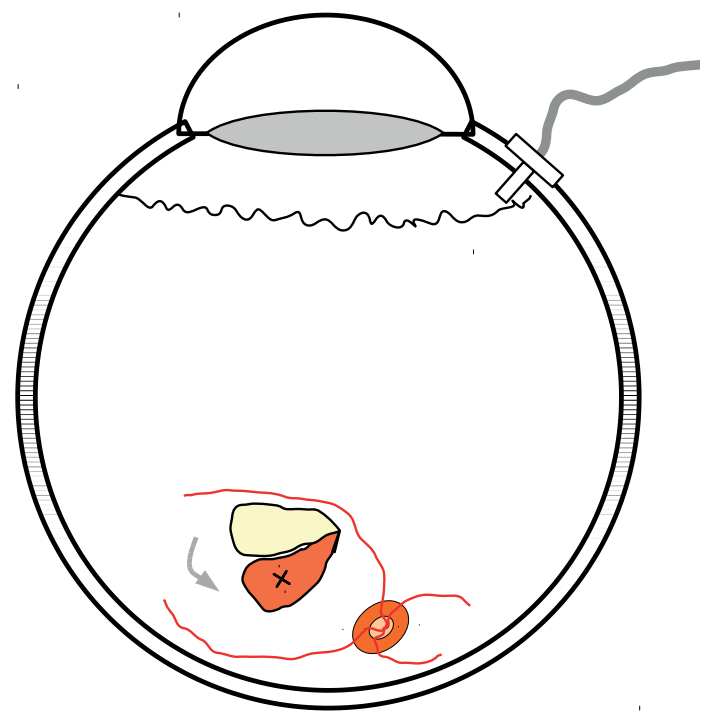

Figure 1. A drawing representing the principle of performing the full thickness pedicled patch of choroid, choriocapillaris, Bruch's membrane and RPE from the superior paramacular uveal bed

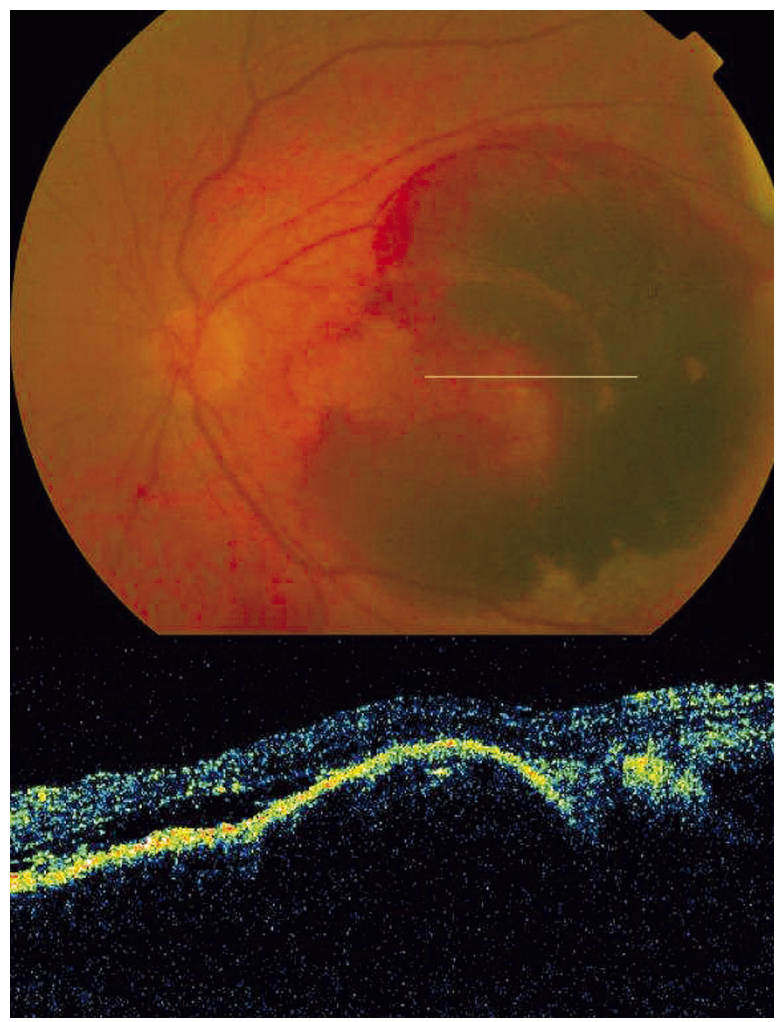

Figure 2. Fundoscopic preoperative image of a 17 DA large subretinal hemorrhage in the macular region and a dome-shaped hemorrhagic sub-RPE elevation with RPE tear and subretinal fluid on OCT 


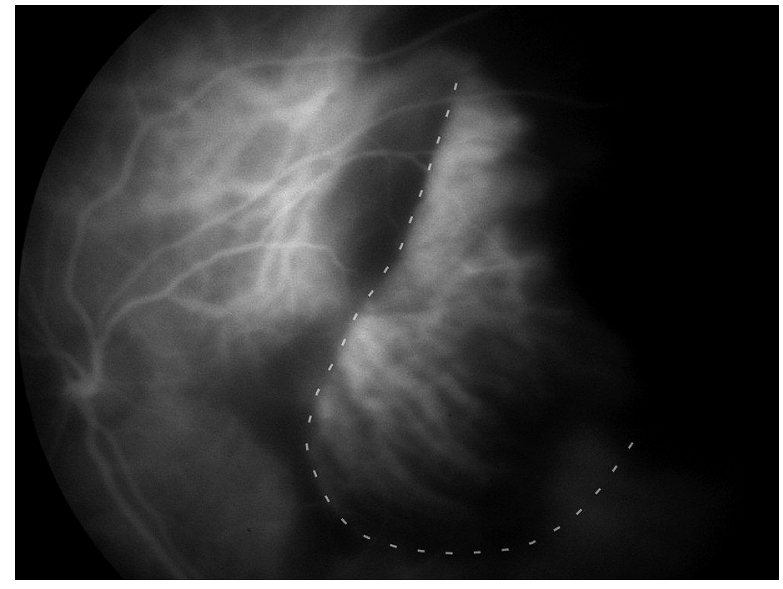

Figure 3. An ICG performed on the third postoperative day. The location of the graft is marked with a dashed line. Perfusion of graft vasculature is visible. The temporal edge of the patch is obscured by mild postoperative bleeding

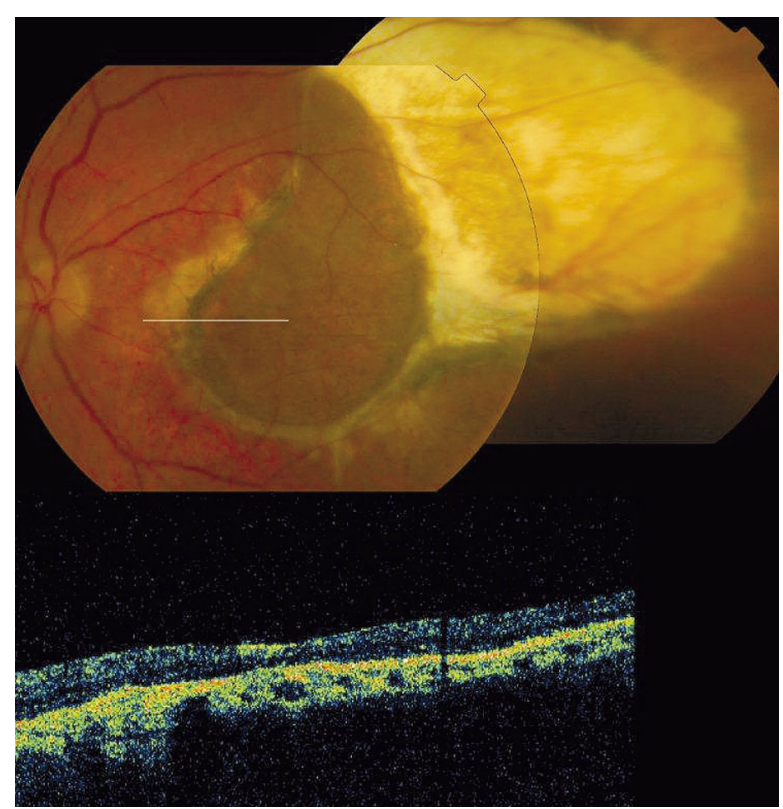

Figure 4. The postoperative fundoscopic image with a pedicled RPE choroid patch at 12 months. The OCT showed a slightly elevated graft with overlying normal thickness retina and foveal contour

No intraoperative complications occurred. The patch was properly positioned under the fovea with an intact pedicle. On the first postoperative day mild hemorrhage from the CNV bed was observed around the graft. Blood was absorbed over the following
3 weeks. On the third postoperative day ICG angiography was performed and showed perfusion through the pedicle and patch vasculature (Figure 3). At the end of the follow-up OCT showed a slightly elevated graft with overlying normal thickness retina with normal foveal contour (Figure 4). No CNV recurrence was observed.

Best corrected visual acuity improved from $0.5 / 50$ to $5 / 50$ at 1 month and remained stable over 1 year of follow-up.

\section{Discussion}

This study demonstrates the feasibility of a full thickness pedicled RPE-choroid graft that is an alternative to a free isolated graft. A pedicle with choroidal vasculature offers a hypothetical advantage of maintaining the perfusion immediately after the surgery.

Blood supply to the graft is critical for a successful outcome. Unfortunately not all isolated grafts become vascularized. Even though MacLaren et al. [7] reported $100 \%$ reperfusion in their long-term followup of 4 patients, Maaijwee et al. [5] observed no signs of perfusion in 2 of 31 patients in their case series and Joussen et al. in 2 of 40 eyes [6]. It is believed that non-perfusion of the patch correlates with low visual acuity after the surgery [5]. We speculate that a pedicle with vessels will allow this complication to be reduced.

Moreover, even if the patch becomes vascularized in the time necessary for this to occur, some additional damage to photoreceptors is probable. There is clinical evidence that when an isolated patch procedure is performed the earliest partial revascularization occurs at 1 week after the surgery and only in a few patients [4]. This observation is additionally supported by animal studies [9]. Furthermore, absence of immediate perfusion might explain the inferior visual acuity results of isolated patch surgery compared to macular translocation surgery [10]. In contrast in our patient the perfusion of the entire graft was observed on the third postoperative day. We believe this contributed to the quick improvement of BCVA to 5/50 in the first month. We did not perform ICG angiography on the first postoperative day but we hypothesize that a pedicled patch actually preserved the perfusion rather than received new feeder vessels. For this reason revascularization is not critical, even though new vessels may augment the perfusion after several weeks. 
In earlier reports Peyman et al. [11] used a narrow pedicled graft of RPE cell sheet. Stanga et al. [12] performed one pedicled full thickness graft in his series of 6 eyes. Nevertheless, they did not examine the early perfusion. Both approached the subretinal space through a small posterior retinotomy. In our opinion this limited manipulation options and capability to select a pedicle containing a large choroid vessel. Transplantation involving a $180^{\circ}$ peripheral retinotomy and folding the temporal retina onto nasal retina $[2,4,13]$ offers an easy and safe access to the CNV and choroid. Application of PFCL on the choroid and retina keeps the retina folded nasally in a safe position. This allows one to remove the CNV and blood and to mark an area chosen for grafting with laser photocoagulation. Our selection was based on the appearance of a healthy RPE that was assessed by the surgeon and the presence of a large choroidal vessel in the pedicle.

In our study bleeding from the CNV bed was observed on the first postoperative day. This is a frequent complication observed in $10-100 \%$ of cases [2, 4, 6, 14-16]. Fortunately, no signs of proliferative vitreoretinopathy were present.

In conclusion, our modification of choroid RPE transplantation surgery, by demonstrating early perfusion offers an advantage, similar to macular translocation, when photoreceptors are embedded in RPE and choroid with blood circulation immediately after the surgery.

The paper was presented at the 11th EURETINA Congress in London on May 29th, 2011.

\section{References}

1. Leibowitz HM, Krueger DE, Maunder LR, et al. The framingham eye study monograph: an ophthalmological and epidemiological study of cataract, glaucoma, diabetic retinopathy, macular degeneration, and visual acuity in a general population of 2631 adults, 1973-1975. Surv Ophthalmol 1980; 24: 335-610.

2. Gibran SK, Romano MR, Wong D. Perfluorocarbon liquid assisted large retinal epithelium patching in sub-macular hemorrhage secondary to age related macular degeneration. Graefes Arch Clin Exp Ophthalmol 2009; 247: 187-91.

3. Maaijwee K, Joussen AM, Kirchhof B, van Meurs JC. Retinal pigment epithelium (RPE)-choroid graft translocation in the treatment of an RPE tear: preliminary results. Br J Ophthalmol 2008; 92: 526-9.

4. Cereda MG, Parolini B, Bellesini E, Pertile G. Surgery for cnv and autologous choroidal rpe patch transplantation: exposing the submacular space. Graefes Arch Clin Exp Ophthalmol 2010; 248: 37-47.
5. Maaijwee K, Van Den Biesen PR, Missotten T, Van Meurs JC. Angiographic evidence for revascularization of an rpe-choroid graft in patients with age-related macular degeneration. Retina (Philadelphia, Pa.) 2008; 28: 498-503.

6. Joussen AM, Heussen FMA, Joeres S, et al. Autologous translocation of the choroid and retinal pigment epithelium in agerelated macular degeneration. Am J Ophthalmol 2006; 142: 17-30.

7. MacLaren RE, Bird AC, Sathia PJ, Aylward GW. Long-term results of submacular surgery combined with macular translocation of the retinal pigment epithelium in neovascular age-related macular degeneration. Ophthalmology 2005; 112: 2081-7.

8. Hallock GG. Classification of flaps. In: Flaps and reconstructive surgery. Wei FC, Mardini S (eds.). Saunders 2009; 7-15.

9. Maaijwee KJM, van Meurs JC, Kirchhof B, et al. Histological evidence for revascularisation of an autologous retinal pigment epithelium: choroid graft in the pig. Br J Ophthalmol 2007; 91: 546-50.

10. Chen FK, Patel PJ, Uppal GS, et al. A comparison of macular translocation with patch graft in neovascular age-related macular degeneration. Invest Ophthalmol Vis Sci 2009; 50: 1848-55.

11. Peyman GA, Blinder KJ, Paris CL, et al. A technique for retinal pigment epithelium transplantation for age-related macular degeneration secondary to extensive subfoveal scarring. Ophthal Surg 1991; 22: 102-8.

12. Stanga PE, Kychenthal A, Fitzke FW, et al. Retinal pigment epithelium translocation and central visual function in age related macular degeneration: preliminary results. Int Ophthalmol 2001; 23: 297-307.

13. Ma Z, Han L, Wang C, et al. Autologous transplantation of retinal pigment epithelium-bruch's membrane complex for hemorrhagic age-related macular degeneration. Invest Ophthalmol Vis Sci 2009; 50: 2975-81.

14. Maaijwee K, Heimann H, Missotten T, et al. Retinal pigment epithelium and choroid translocation in patients with exudative age-related macular degeneration: long-term results. Graefes Arch Clin Exp Ophthalmol 2007; 245: 1681-9.

15. MacLaren RE, Uppal GS, Balaggan KS, et al. Autologous transplantation of the retinal pigment epithelium and choroid in the treatment of neovascular age-related macular degeneration. Ophthalmology 2007; 114: 561-70.

16. van Meurs JC, Van Den Biesen PR. Autologous retinal pigment epithelium and choroid translocation in patients with exudative age-related macular degeneration: short-term follow-up. Am J Ophthalmol 2003; 136: 688-95. 